Susumu Saito $\cdot$ Aritoshi Iida $\cdot$ Akihiro Sekine

Chie Ogawa · Saori Kawauchi · Shoko Higuchi

Yusuke Nakamura

\title{
Catalog of 238 variations among six human genes encoding solute carriers (hSLCs) in the Japanese population
}

Received: July 24, 2002 / Accepted: July 25, 2002

\begin{abstract}
We screened DNAs of 48 Japanese individuals for single-nucleotide polymorphisms (SNPs) in six genes encoding proteins of the solute carrier (SLC) family by direct sequencing of their entire genomic regions except for repetitive-sequence elements. This approach identified 213 SNPs and 25 insertion/deletion polymorphisms among the six genes. On average, we identified 1 SNP in every 509 nucleotides. Of the $213 \mathrm{SNPs}, 14$ were identified in the $S L C 10 A 1$ gene, 51 in SLC15A1, 29 in SLC22A1, 27 in $S L C 22 A 2,54$ in $S L C 22 A 4$, and 38 in SLC22A5. Eight were located in $5^{\prime}$ flanking regions, 172 in introns, 25 in exons, and 8 in $3^{\prime}$ flanking regions. These variants should contribute to investigations of possible correlations between genotypes and phenotypes as regards disease susceptibilities or responsiveness to drug therapy.
\end{abstract}

Key words SNP - SLC10A1 - SLC15A1 - SLC22A1 · SLC22A $2 \cdot$ SLC22A $4 \cdot$ SLC22A 5

\section{Introduction}

Member 1 of human solute carrier (SLC) family 10 (sodium/bile acid cotransporters), SLC10A1 (NTCP1), is

S. Saito $\cdot$ A. Iida $\cdot$ A. Sekine $\cdot$ S. Kawauchi $\cdot$ S. Higuchi $\cdot$

Y. Nakamura

Laboratory for Genotyping, SNP Research Center, Yokohama Institute, Institute of Physical and Chemical Research, Yokohama, Japan

C. Ogawa · Y. Nakamura $(\bowtie)$

Laboratory of Molecular Medicine, Human Genome Center,

Institute of Medical Science, The University of Tokyo, 4-6-1

Shirokanedai, Minato-ku, Tokyo 108-8639, Japan

Tel. +81-3-5449-5372; Fax +81-3-5449-5433

e-mail: yusuke@ims.u-tokyo.ac.jp the major transport protein responsible for sodiumdependent uptake of bile salts by hepatocytes from the portal circulation (Meier 1995). This protein is localized specifically to liver sinusoidal membranes (Craddock et al. 1998; Muller and Jansen 1997). The promoter region of the gene encoding SLC10A1 contains potential DNA-binding sites for transcription factors $\mathrm{C} / \mathrm{EBP}$ and HNF3/HNF6, and in some experiments mutations of these C/EBP sites have resulted in loss of $77 \%$ of transcriptional activity (Shiao et al. 2000). Expression of this gene is markedly reduced in several experimental rodent models exhibiting cholestasis (Gartung et al. 1996; Green et al. 1997).

Member 1 (SLC15A1, PEPT1) of human solute carrier family 15 (oligopeptide transporters) is located in the intestinal brush border membrane (Leibach and Ganapathy 1996). SLC15A1 mediates absorption of dipeptides and tripeptides that arise from the digestion of dietary proteins or beta-lactam antibiotics and other peptide-like drugs (Adibi 1997). This protein contains 12 predicted transmembrane domains (TMDs), and a large extracellular loop carries potential $\mathrm{N}$-glycosylation sites (Liang et al. 1995; Covitz et al. 1998).

A newly described transporter family (solute carrier family 22; SLC22) consists of organic cation transporters (OCTs). OCTs function primarily to eliminate cationic drugs and other xenobiotics from tissues such as kidney, small intestine, and liver (Koepsell 1998; Zhang et al. 1998). Member 1 of family 22 (SLC22A1, OCT1) is transcribed primarily in the liver (Gorboulev et al. 1997; Zhang et al. 1997). However, member 2 (SLC22A2, OCT2) is expressed mainly in the kidney (Gorboulev et al. 1997). Member 4 (SLC22A4, OCTN1) is a renal proton/organic cation antiporter functioning at the epithelial apical membrane (Tamai et al. 1997; Yabuuchi et al. 1999); it is strongly expressed in kidney, trachea, bone marrow, and fetal liver, but not in adult liver. Member 5 (SLC22A5, OCTN2) is strongly expressed in kidney, skeletal muscle, heart, and placenta in adult humans (Tamai et al. 1998); mutations in the $S L C 22 A 5$ gene cause carnitine deficiency (Lamhonwah and Tein 1998; Burwinkel et al. 1999; Nezu et al. 1999; Tang et al. 1999; Vaz et al. 1999; Wang et al. 1999, 2000, 2001). All 
these transporters have 11 (SLC22A4) or 12 (SLC22A1, SLC22A2, and SLC22A5) TMDs, and each has a large extracellular loop carrying potential $\mathrm{N}$-glycosylation sites (Tamai et al. 1997; Burckhardt and Wolff 2000). Conserved amino acid motifs reveal a relationship to the sugar transport-facilitator family of molecules (Burckhardt and Wolff 2000).

To investigate in more detail the nature of apparent genotype/phenotype correlations for some SLCs, we began by searching for additional single-nucleotide polymorphisms (SNPs) in the six genes encoding the transporters described above, including their promoter regions and introns except for repetitive elements, and report here a total of 238 genetic variations, of which 129 have not been reported before.

\section{Subjects and methods}

Total genomic DNAs were isolated from peripheral leukocytes of 48 unrelated Japanese individuals by the standard phenol/chloroform extraction method, after informed consent was obtained from each participant. On the basis of sequence information from GenBank we designed polymerase chain reaction (PCR) primers to amplify DNA fragments from all six $S L C$ genes, excluding repetitive elements, by invoking the REPEAT MASKER computer program (http://ftp.genome.washington.edu/cgi-bin/ RepeatMasker). PCR experiments and DNA sequencing were performed according to methods described previously (Iida et al. 2001; Saito et al. 2001; Sekine et al. 2001). All SNPs detected by the PolyPhred computer program
(Nickerson et al. 1997) were confirmed by sequencing both strands of each PCR product.

\section{Results}

Exon-intron boundaries were defined by comparison of genomic sequences with cDNA sequences; all accession numbers are listed in Table 1. We screened 96 Japanese chromosomes for SNPs in these SLC genes by means of direct DNA sequencing.

Subsequent resequencing of a total of about $109 \mathrm{~kb}$ of genomic DNA $(13.2 \mathrm{~kb}$ for the $S L C 10 A 1$ gene, $23 \mathrm{~kb}$ for $S L C 15 A 1,17 \mathrm{~kb}$ for $S L C 22 A 1,13.4 \mathrm{~kb}$ for $S L C 22 A 2$, $22.9 \mathrm{~kb}$ for $S L C 22 A 4$, and $19 \mathrm{~kb}$ for $S L C 22 A 5)$ identified 213 SNPs and 25 insertion/deletion polymorphisms (Table 2). Figure 1a-f illustrates the location of each variation we found; detailed information on nucleotide positions and substitutions is summarized in Table $3 a-f$. On average, we identified $1 \mathrm{SNP}$ in every 509 nucleotides. Of the 238 genetic variations identified in our screening (including insertion/ deletion polymorphisms), 129 (54\%) were not reported previously.

Among the 213 SNPs mapped in our experiments, 8 were located in $5^{\prime}$ flanking regions, 172 in introns, 25 in exons, and 8 in $3^{\prime}$ flanking regions (Table 4). Among the 25 SNPs detected in exons, 1 was located in a $5^{\prime}$ UTR, 18 were in coding regions, and 6 were in $3^{\prime}$ UTRs. Ten of the 18 SNPs detected in the coding regions would cause substitution of amino acids and 4 of those had not been reported before (Gly419Ala in SLC15A1, Pro283Leu and Arg287Gly in $S L C 22 A 1$, and Gly462Glu in SLC22A4) (Table 5).

Table 1. Accession numbers for the genomic and cDNA sequences used in this study

\begin{tabular}{|c|c|c|c|c|}
\hline \multirow[b]{2}{*}{ Gene name } & \multirow[b]{2}{*}{ Chromosomal localization } & \multicolumn{3}{|l|}{ Accession number } \\
\hline & & Genomic sequence & & cDNA sequence \\
\hline SLC10A1 & $14 \mathrm{q} 24.1$ & AL157789.6 & & XM_007466.3 \\
\hline$S L C 15 A 1$ & $13 q 33-q 34$ & AL353574.8 & AL391670.6 & NM_005073.1 \\
\hline$S L C 22 A 1$ & $6 q 26$ & AL353625.5 & & X98332.1 \\
\hline$S L C 22 A 2$ & $6 q 26$ & AL162582.18 & & NM_003058.1 \\
\hline$S L C 22 A 4$ & $5 q 31.1$ & AC008599.6 & & Y09881.1 \\
\hline$S L C 22 A 5$ & $5 q 31$ & AC023861.3 & AB016625.1 & XM_003701.3 \\
\hline
\end{tabular}

Table 2. Summary of genetic variations in six $S L C$ genes

\begin{tabular}{|c|c|c|c|c|c|c|}
\hline Gene & $\begin{array}{l}\text { All genetic } \\
\text { variations }\end{array}$ & SNPs & $\begin{array}{l}\text { Insertion/deletion } \\
\text { polymorphisms }\end{array}$ & Novel & $\begin{array}{l}\text { Total base pairs } \\
\text { sequenced }(\mathrm{kb})\end{array}$ & $\begin{array}{l}\text { Frequency } \\
\text { (bp/1SNP) }\end{array}$ \\
\hline SLC10A1 & 18 & 14 & 4 & 15 & 13.2 & 943 \\
\hline SLC15A1 & 57 & 51 & 6 & 38 & 23.0 & 451 \\
\hline SLC $22 A 1$ & 33 & 29 & 4 & 22 & 17.0 & 586 \\
\hline$S L C 22 A 2$ & 32 & 27 & 5 & 12 & 13.4 & 496 \\
\hline$S L C 22 A 4$ & 58 & 54 & 4 & 30 & 22.9 & 424 \\
\hline$S L C 22 A 5$ & 40 & 38 & 2 & 12 & 19.0 & 500 \\
\hline Total & 238 & 213 & 25 & 129 & 108.5 & (average) 509 \\
\hline
\end{tabular}

SNP, Single-nucleotide polymorphism; SLC, solute carrier 
a Solute carrier family 10, member 1 (SLC10A1)

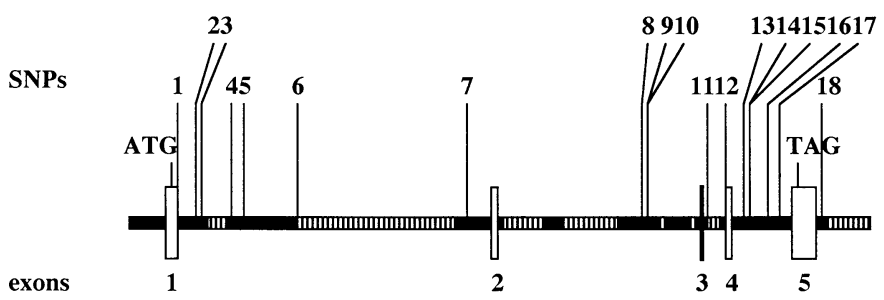

b Solute carrier family 15, member 1 (SLC15A1)
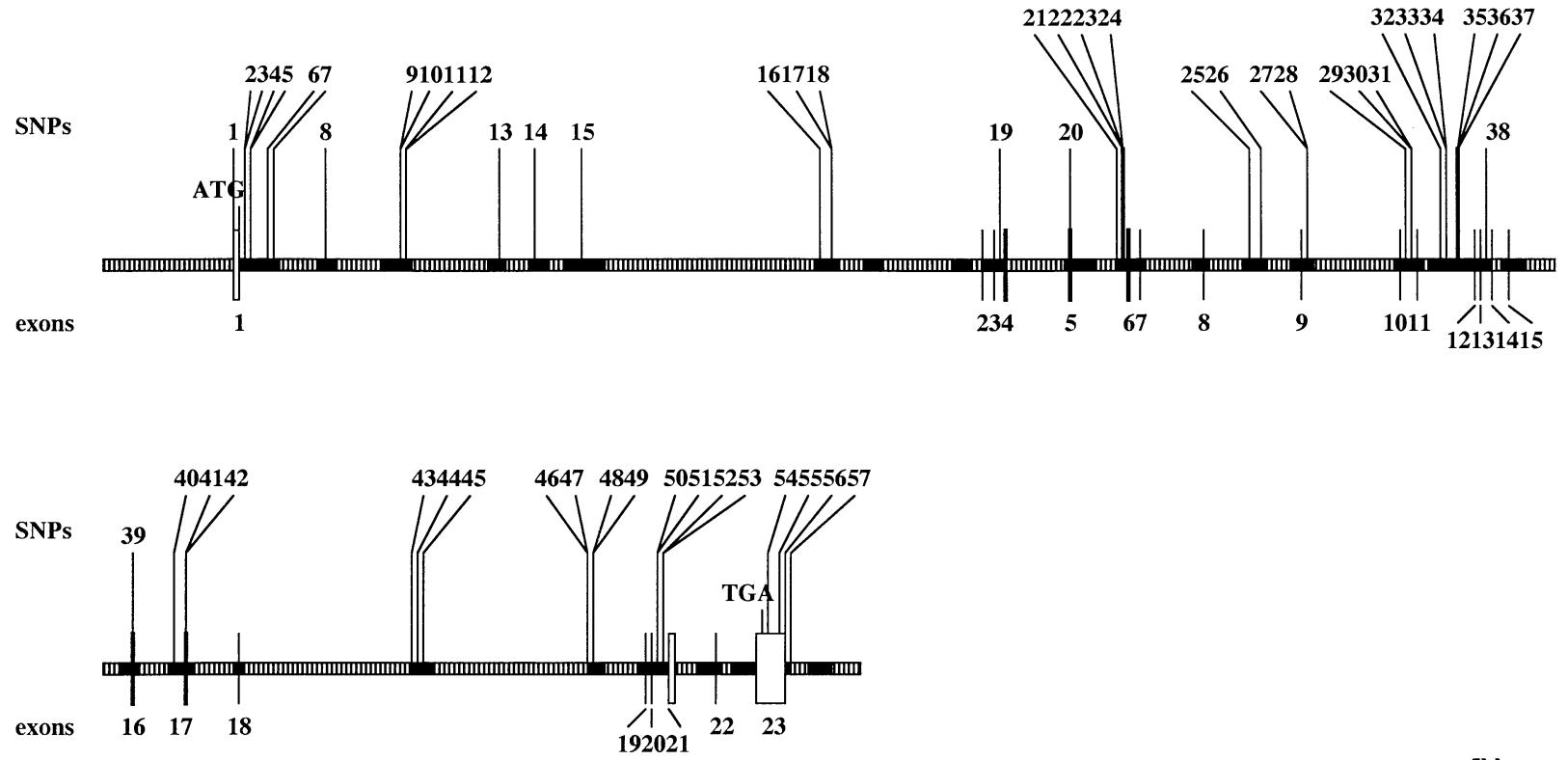

c Solute carrier family 22 , member 1 (SLC22A1)

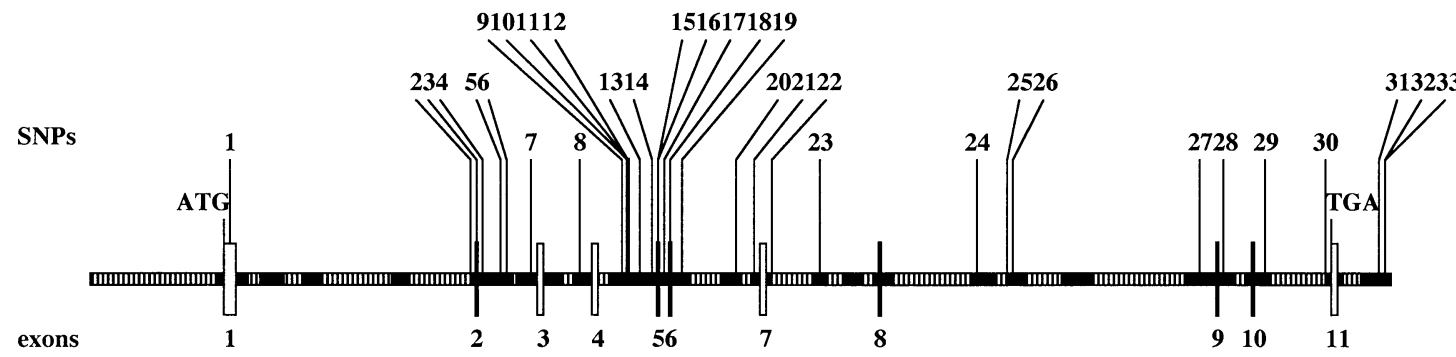

$5 \mathrm{~kb}$

Fig. 1. Locations of single-nucleotide polymorphisms (SNPs) in the SLC10A1 (a), SLC15A1 (b), SLC22A1 (c), SLC22A2 (d), SLC22A4 (e), and $S L C 22 A 5$ (f) genes, indicated by vertical lines. Open boxes represent exons; hatching on the chromosomes indicates unsequenced repetitive elements. $A T G$, initiation codon; $T G A, T A G$, or $T A A$, stop codons 
d Solute carrier family 22, member 2 (SLC22A2)

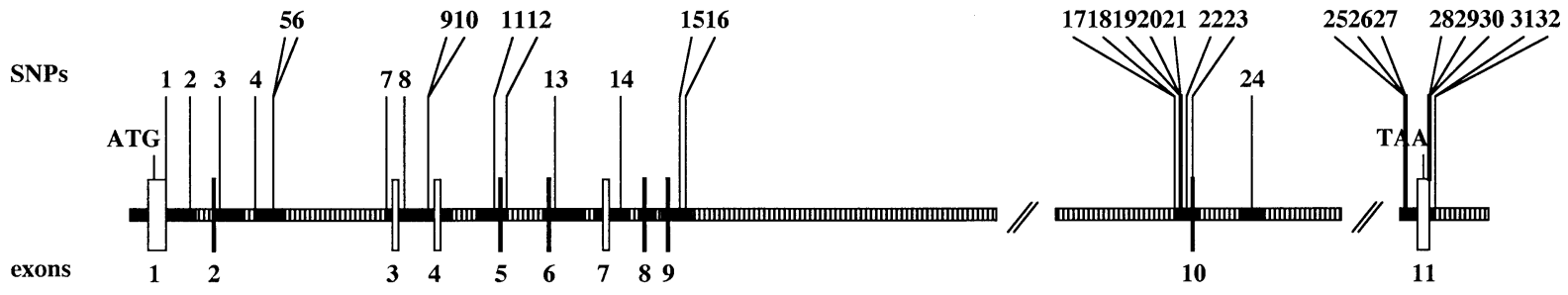

e Solute carrier family 22, member 4 (SLC22A4)

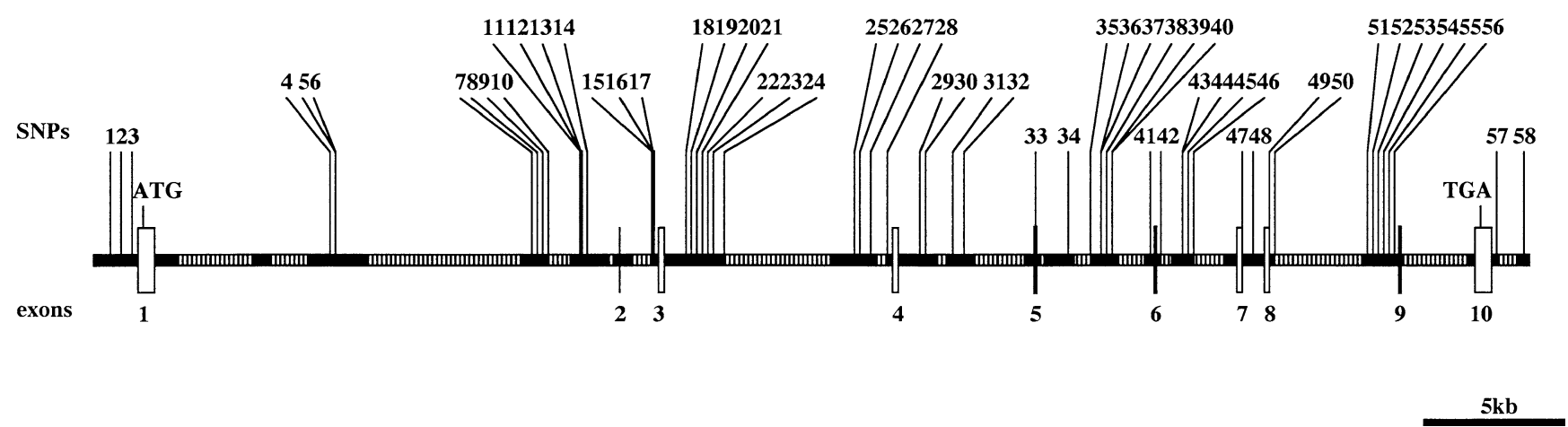

f Solute carrier family 22, member 5 (SLC22A5)

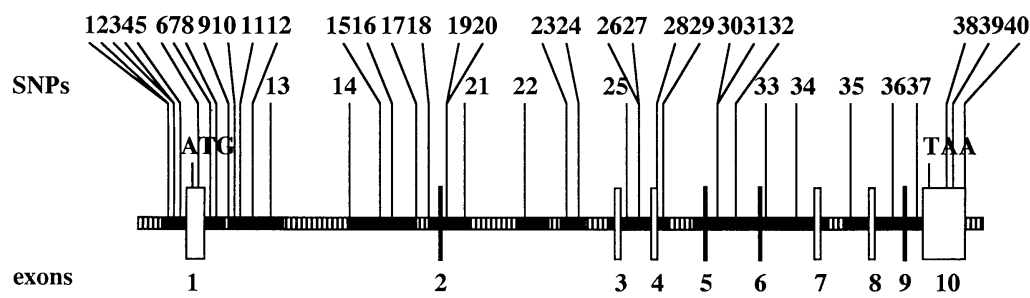

Fig. 1a-f. Continued 
Table 3a. Summary of genetic variations detected in the SLC10A1 gene

\begin{tabular}{|c|c|c|c|c|c|c|c|c|c|}
\hline No. & Location & Position $^{\mathrm{a}}$ & Genetic variation & NCBI SNP ID & No. & Location & Position $^{\mathrm{a}}$ & Genetic variation & NCBI SNP ID \\
\hline 1 & Exon 1 & 307 & G/A(Thr75Thr) & & 10 & Intron 2 & 5046 & $\mathrm{~A} / \mathrm{T}$ & \\
\hline 2 & Intron 1 & 607 & $\mathrm{G} / \mathrm{C}$ & & 11 & Intron 3 & $(8-21)$ & $(\mathrm{T})_{12-15}$ & \\
\hline 3 & Intron 1 & 702 & $\mathrm{G} / \mathrm{A}$ & & 12 & Exon 4 & 54 & $\mathrm{C} / \mathrm{T}$ (Ser267Phe) & rs2296651 \\
\hline 4 & Intron 1 & 2192 & $\mathrm{C} / \mathrm{T}$ & rs943277 & 13 & Intron 4 & $(484-495)$ & $(\mathrm{A})_{10-13}$ & \\
\hline 5 & Intron 1 & 2587 & $\mathrm{C} / \mathrm{T}$ & rs943276 & 14 & Intron 4 & $(728-754)$ & $(\mathrm{A})_{25-27}$ & \\
\hline 6 & Intron 1 & $(3950-3966)$ & $(\mathrm{T})_{14-17}$ & & 15 & Intron 4 & 747 & $\mathrm{~A} / \mathrm{C}$ & \\
\hline 7 & Intron 1 & 9597 & $\mathrm{C} / \mathrm{G}$ & & 16 & Intron 4 & 1339 & $\mathrm{C} / \mathrm{A}$ & \\
\hline 8 & Intron 2 & 4808 & $\mathrm{C} / \mathrm{T}$ & & 17 & Intron 4 & 1545 & $\mathrm{G} / \mathrm{C}$ & \\
\hline 9 & Intron 2 & 5032 & $\mathrm{G} / \mathrm{C}$ & & 18 & 3' Flanking & 559 & G/A & \\
\hline
\end{tabular}

SLC10A1, Solute carrier family 10, member 1; NCBI, National Center for Biotechnology Information; UTR, untranslated region; del, deletion; ins, insertion

${ }^{a}$ For SNPs in the 5' flanking region, intron, or 3' flanking region, nucleotide positions are counted from the first intronic nucleotide at the exon/ intron junction (for SNPs in the exon, nucleotide positions are counted from the first exonic nucleotide at the exon/intron junction)

Table 3b. Summary of genetic variations detected in the SLC15A1 gene

\begin{tabular}{|c|c|c|c|c|c|c|c|c|c|}
\hline No. & Location & Position $^{a}$ & Genetic variation & NCBI SNP ID & No. & Location & Position $^{\mathrm{a}}$ & Genetic variation & NCBI SNP ID \\
\hline 1 & Exon 1 & 25 & C/T(5'UTR) & & 30 & Intron 10 & 388 & $\mathrm{C} / \mathrm{T}$ & \\
\hline 2 & Intron 1 & 88 & $\mathrm{G} / \mathrm{A}$ & & 31 & Intron 10 & 457 & $\mathrm{~A} / \mathrm{G}$ & rs1289393 \\
\hline 3 & Intron 1 & 106 & $\mathrm{~A} / \mathrm{T}$ & & 32 & Intron 11 & 720 & $\mathrm{G} / \mathrm{A}$ & rs1289392 \\
\hline 4 & Intron 1 & 248 & $\mathrm{G} / \mathrm{A}$ & & 33 & Intron 11 & 985 & $\mathrm{C} / \mathrm{T}$ & \\
\hline 5 & Intron 1 & 326 & $\mathrm{C} / \mathrm{A}$ & & 34 & Intron 11 & $(1022-1045)$ & $(\mathrm{T})_{20-24}$ & \\
\hline 6 & Intron 1 & 1147 & $\mathrm{C} / \mathrm{A}$ & rs914300 & 35 & Intron 11 & 1234 & $\mathrm{C} / \mathrm{T}$ & rs1892503 \\
\hline 7 & Intron 1 & 1238 & $\mathrm{C} / \mathrm{T}$ & & 36 & Intron 11 & 1285 & $\mathrm{~T} / \mathrm{C}$ & rs1289391 \\
\hline 8 & Intron 1 & 3001 & $\mathrm{C} / \mathrm{T}$ & & 37 & Intron 11 & 1320 & $\mathrm{C} / \mathrm{T}$ & \\
\hline 9 & Intron 1 & 5673 & $\mathrm{G} / \mathrm{C}$ & & 38 & Intron 13 & 37 & $\mathrm{C} / \mathrm{T}$ & rs2297319 \\
\hline 10 & Intron 1 & 5679 & $\mathrm{C} / \mathrm{G}$ & & 39 & Exon 16 & 107 & G/C(Gly419Ala) & \\
\hline 11 & Intron 1 & 5917 & $\mathrm{C} / \mathrm{T}$ & & 40 & Intron 16 & 1414 & $\mathrm{~T} / \mathrm{C}$ & rs1756988 \\
\hline 12 & Intron 1 & 5966 & $\mathrm{C} / \mathrm{T}$ & & 41 & Exon 17 & 78 & T/C(Ala449Ala) & rs1339067 \\
\hline 13 & Intron 1 & 9255 & $\mathrm{~A} / \mathrm{G}$ & & 42 & Exon 17 & 79 & G/A(Val459Ile) & rs 2274828 \\
\hline 14 & Intron 1 & 10278 & $\mathrm{~A} / \mathrm{G}$ & & 43 & Intron 18 & 6048 & $\mathrm{~T} / \mathrm{del}$ & \\
\hline 15 & Intron 1 & 11945 & $\mathrm{~T} / \mathrm{C}$ & rs1331251 & 44 & Intron 18 & $(6141-6142)$ & T/ins & \\
\hline 16 & Intron 1 & 20251 & $\mathrm{C} / \mathrm{T}$ & & 45 & Intron 18 & $(6241-6242)$ & G/ins & \\
\hline 17 & Intron 1 & 20509 & $\mathrm{C} / \mathrm{A}$ & & 46 & Intron 18 & 12102 & $\mathrm{C} / \mathrm{T}$ & \\
\hline 18 & Intron 1 & 20532 & $\mathrm{~T} / \mathrm{C}$ & & 47 & Intron 18 & 12104 & $\mathrm{G} / \mathrm{A}$ & rs 2802393 \\
\hline 19 & Intron 3 & 55 & $\mathrm{C} / \mathrm{del}$ & & 48 & Intron 18 & 12203 & $\mathrm{C} / \mathrm{A}$ & \\
\hline 20 & Exon 5 & 105 & G/A(Ser117Asn) & rs2297322 & 49 & Intron 18 & 12307 & $\mathrm{~A} / \mathrm{G}$ & \\
\hline 21 & Intron 5 & 1720 & $\mathrm{C} / \mathrm{A}$ & & 50 & Intron 20 & 12 & G/A & rs2297318 \\
\hline 22 & Intron 5 & 1790 & G/A & & 51 & Intron 20 & 79 & $\mathrm{~A} / \mathrm{G}$ & \\
\hline 23 & Intron 5 & 1860 & $\mathrm{G} / \mathrm{A}$ & & 52 & Intron 20 & 417 & $\mathrm{C} / \mathrm{T}$ & rs950906 \\
\hline 24 & Intron 5 & 1943 & $\mathrm{G} / \mathrm{A}$ & & 53 & Intron 20 & 465 & $\mathrm{~A} / \mathrm{G}$ & rs950905 \\
\hline 25 & Intron 8 & 1478 & $\mathrm{~A} / \mathrm{G}$ & & 54 & Exon 23 & $(348-370)$ & $(\mathrm{T})_{18-23}\left(3^{\prime} \mathrm{UTR}\right)$ & \\
\hline 26 & Intron 8 & 1898 & $\mathrm{~A} / \mathrm{G}$ & & 55 & Exon 23 & 790 & A/G(3'UTR) & \\
\hline 27 & Intron 9 & 149 & $\mathrm{C} / \mathrm{G}$ & rs2297321 & 56 & Exon 23 & 880 & G/A(3'UTR) & rs1289389 \\
\hline 28 & Intron 9 & 221 & $\mathrm{G} / \mathrm{A}$ & rs2297320 & 57 & 3' Flanking & 2 & $\mathrm{G} / \mathrm{A}$ & \\
\hline 29 & Intron 10 & 153 & $\mathrm{~T} / \mathrm{G}$ & rs1289394 & & & & & \\
\hline
\end{tabular}

SLC15A1, Solute carrier family 15, member 1

${ }^{\text {a }}$ For SNPs in the 5' flanking region, intron, or 3' flanking region, nucleotide positions are counted from the first intronic nucleotide at the exon/ intron junction (for SNPs in the exon, nucleotide positions are counted from the first exonic nucleotide at the exon/intron junction)

\section{Discussion}

We identified 238 genetic variations $(213$ SNPs and 25 insertion/deletion polymorphisms) by screening the entire genomic regions, except for repetitive sequences, containing six SLC genes (SLC10A1, SLC15A1, SLC22A1, $S L C 22 A 2, S L C 22 A 4$ and $S L C 22 A 5)$ in 96 chromosomes from 48 unrelated Japanese individuals. The polymor- phisms published here should be useful for examining relationships between genotypes and susceptibilities to certain diseases or the efficacy or adverse effects of certain drugs.

The promoter region of the $S L C 10 A 1$ gene contains potential DNA-binding sites for transcription factors C/EBP and HNF3/HNF6 (Shiao et al. 2000). Although mutations at the C/EBP sites markedly reduce transcriptional activity (Shiao et al. 2000), we found no variations in this region in our test population. 
Table 3c. Summary of genetic variations detected in the $S L C 22 A 1$ gene

\begin{tabular}{|c|c|c|c|c|c|c|c|c|c|}
\hline No. & Location & Position $^{\mathrm{a}}$ & Genetic variation & NCBI SNP ID & No. & Location & Position $^{a}$ & Genetic variation & NCBI SNP ID \\
\hline 1 & Exon 1 & 228 & $\mathrm{~T} / \mathrm{C}($ Ser52Ser $)$ & rs1867351 & 18 & Exon 6 & 68 & C/T(Pro341Leu) & rs2282143 \\
\hline 2 & Intron 1 & 7715 & $\mathrm{G} / \mathrm{T}$ & & 19 & Intron 6 & 379 & $\mathrm{~A} / \mathrm{G}$ & \\
\hline 3 & Exon 2 & 69 & C/G(Phe160Leu) & rs683369 & 20 & Intron 6 & 2125 & $\mathrm{C} / \mathrm{A}$ & \\
\hline 4 & Intron 2 & 97 & $\mathrm{G} / \mathrm{A}$ & & 21 & Intron 6 & $(2935-2953)$ & $(\mathrm{T})_{18-20}$ & \\
\hline 5 & Intron 2 & 797 & $\mathrm{C} / \mathrm{G}$ & & 22 & Intron 7 & $(6-7)$ & TGGTAAGT/ins & \\
\hline 6 & Intron 2 & 1015 & $\mathrm{~T} / \mathrm{G}$ & rs614890 & 23 & Intron 7 & $(1780-1781)$ & T/ins & \\
\hline 7 & Intron 2 & 1768 & $\mathrm{~T} / \mathrm{C}$ & & 24 & Intron 8 & 3247 & $\mathrm{G} / \mathrm{T}$ & \\
\hline 8 & Intron 3 & 1244 & $\mathrm{C} / \mathrm{T}$ & & 25 & Intron 8 & 4215 & $\mathrm{~T} / \mathrm{C}$ & rs654993 \\
\hline 9 & Intron 4 & 865 & $\mathrm{G} / \mathrm{T}$ & & 26 & Intron 8 & 4316 & $\mathrm{G} / \mathrm{A}$ & rs2197296 \\
\hline 10 & Intron 4 & 990 & $\mathrm{C} / \mathrm{T}$ & rs806383 & 27 & Intron 8 & 10521 & G/A & \\
\hline 11 & Intron 4 & 1028 & $\mathrm{~A} / \mathrm{G}$ & & 28 & Intron 9 & 43 & $\mathrm{C} / \mathrm{T}$ & rs2297374 \\
\hline 12 & Intron 4 & 1040 & $\mathrm{~T} / \mathrm{G}$ & & 29 & Intron 10 & 393 & $\mathrm{G} / \mathrm{C}$ & \\
\hline 13 & Intron 4 & 1485 & $\mathrm{C} / \mathrm{T}$ & & 30 & Intron 10 & 2421 & $\mathrm{C} / \mathrm{T}$ & rs622591 \\
\hline 14 & Intron 4 & 1997 & $\mathrm{G} / \mathrm{A}$ & & 31 & 3' Flanking & 1627 & $\mathrm{G} / \mathrm{A}$ & rs651164 \\
\hline 15 & Exon 5 & 9 & C/T(Pro283Leu) & & 32 & 3' Flanking & 1755 & $\mathrm{~T} / \mathrm{C}$ & \\
\hline 16 & Exon 5 & 20 & C/G(Arg287Gly) & & 33 & 3' Flanking & 1799 & G/del & \\
\hline 17 & Intron 5 & 149 & $\mathrm{G} / \mathrm{A}$ & rs2282142 & & & & & \\
\hline
\end{tabular}

SLC22A1, Solute carrier family 22, member 1

${ }^{a}$ For SNPs in the 5' flanking region, intron, or $3^{\prime}$ flanking region, nucleotide positions are counted from the first intronic nucleotide at the exon/ intron junction (for SNPs in the exon, nucleotide positions are counted from the first exonic nucleotide at the exon/intron junction)

Table 3d. Summary of genetic variations detected in the $S L C 22 A 2$ gene

\begin{tabular}{|c|c|c|c|c|c|c|c|c|c|}
\hline No. & Location & Position $^{\mathrm{a}}$ & Genetic variation & NCBI SNP ID & No. & Location & Position $^{\mathrm{a}}$ & Genetic variation & NCBI SNP ID \\
\hline 1 & Exon 1 & 534 & G/T(Thr130Thr) & rs624249 & 17 & Intron 9 & -529 & $\mathrm{~A} / \mathrm{T}$ & rs316006 \\
\hline 2 & Intron 1 & 883 & $\mathrm{~A} / \mathrm{C}$ & rs638360 & 18 & Intron 9 & -444 & $\mathrm{G} / \mathrm{A}$ & rs316005 \\
\hline 3 & Intron 2 & 32 & $\mathrm{C} / \mathrm{G}$ & rs 2774230 & 19 & Intron 9 & -396 & $\mathrm{~A} / \mathrm{G}$ & \\
\hline 4 & Intron 2 & 1329 & $\mathrm{G} / \mathrm{del}$ & & 20 & Intron 9 & -386 & $\mathrm{~A} / \mathrm{C}$ & \\
\hline 5 & Intron 2 & 1867 & $\mathrm{C} / \mathrm{del}$ & & 21 & Intron 9 & -337 & $\mathrm{~A} / \mathrm{C}$ & rs316004 \\
\hline 6 & Intron 2 & 1882 & $\mathrm{G} / \mathrm{A}$ & rs316009 & 22 & Intron 9 & -86 & A/del & \\
\hline 7 & Intron 2 & 5909 & $\mathrm{C} / \mathrm{A}$ & rs 152275 & 23 & Exon 10 & 5 & G/A(Val502Val) & rs316003 \\
\hline 8 & Intron 3 & 80 & $\mathrm{G} / \mathrm{A}$ & rs316016 & 24 & Intron 10 & 1725 & $\mathrm{G} / \mathrm{A}$ & \\
\hline 9 & Intron 3 & 1047 & $\mathrm{G} / \mathrm{A}$ & rs316017 & 25 & Intron 10 & -225 & $\mathrm{~A} / \mathrm{T}$ & rs2450973 \\
\hline 10 & Intron 3 & 1086 & $\mathrm{C} / \mathrm{T}$ & rs316018 & 26 & Intron 10 & -215 & $\mathrm{C} / \mathrm{T}$ & rs2450974 \\
\hline 11 & Intron 4 & 1859 & $\mathrm{~T} / \mathrm{C}$ & rs2279463 & 27 & Intron 10 & -195 & $\mathrm{C} / \mathrm{T}$ & \\
\hline 12 & Intron 5 & 175 & $\mathrm{G} / \mathrm{A}$ & rs316021 & 28 & Exon 11 & 328 & T/del(3'UTR) & \\
\hline 13 & Intron 6 & 115 & $\mathrm{G} / \mathrm{C}$ & rs167217 & 29 & Exon 11 & 427 & $\mathrm{~A} / \mathrm{T}\left(3^{\prime} \mathrm{UTR}\right)$ & \\
\hline 14 & Intron 7 & 421 & $\mathrm{C} / \mathrm{T}$ & rs315993 & 30 & Exon 11 & 455 & G/A(3'UTR $)$ & \\
\hline 15 & Intron 9 & $(340-343)$ & CTCT/del & & 31 & 3' Flanking & 34 & T/A & \\
\hline 16 & Intron 9 & 363 & $\mathrm{~T} / \mathrm{G}$ & rs315990 & 32 & 3' Flanking & 62 & $\mathrm{~A} / \mathrm{C}$ & rs2450975 \\
\hline
\end{tabular}

SLC22A2, Solute carrier family 22, member 2

${ }^{a}$ For SNPs in the $5^{\prime}$ flanking region, intron, or $3^{\prime}$ flanking region, nucleotide positions are counted from the first intronic nucleotide at the exon/ intron junction (for SNPs in the exon, nucleotide positions are counted from the first exonic nucleotide at the exon/intron junction)

Among the four novel nonsynonymous polymorphisms, one, Gly419Ala in SLC15A1, was located in the large extracellular loop that carries potential $\mathrm{N}$-glycosylation sites (Liang et al. 1995; Covitz et al. 1998). On the other hand, two polymorphisms in SLC22A1, Pro283Leu and Arg287Gly, were located in the intracellular loop that carries potential phosphorylation sites for protein kinase $\mathrm{C}$ (Zhang et al. 1997). The fourth, Gly462Glu, was located in a TMD of SLC22A4. Any of these four polymorphisms could have phenotypic consequences.

Comparison of amino acids in the four organic cation transporters (SLC22A1, SLC22A2, SLC22A4, and
SLC22A5) revealed conservation of 4 cysteines (Cys), 13 prolines (Pro), 3 aspartic acids (Asp), 6 glutamic acids (Glu), and 7 arginines (Arg). These residues are thought to maintain secondary structures of proteins and/or bind charged substrates (Burckhardt and Wolff 2000). The two nonsynonymous SNPs in SLC22A1, Pro283Leu and Arg287Gly, involved conserved residues, suggesting that these polymorphisms could be associated with altered susceptibility to certain diseases.

All data for the genetic variations reported here are available through our website (http://snp.ims.utokyo.ac.jp/). 
Table 3e. Summary of genetic variations detected in the SLC22A4 gene

\begin{tabular}{|c|c|c|c|c|c|c|c|c|c|}
\hline No. & Location & Position $^{\mathrm{a}}$ & Genetic variation & NCBI SNP ID & No. & Location & Position $^{\mathrm{a}}$ & Genetic variation & NCBI SNP ID \\
\hline 1 & $5^{\prime}$ Flanking & -987 & $\mathrm{~A} / \mathrm{G}$ & rs162887 & 30 & Intron 4 & 1055 & $\mathrm{G} / \mathrm{A}$ & \\
\hline 2 & 5' Flanking & -380 & $\mathrm{C} / \mathrm{G}$ & rs460089 & 31 & Intron 4 & 2071 & $\mathrm{G} / \mathrm{C}$ & rs273915 \\
\hline 3 & $5^{\prime}$ Flanking & -90 & $\mathrm{G} / \mathrm{C}$ & rs460271 & 32 & Intron 4 & 2383 & $\mathrm{~A} / \mathrm{T}$ & rs273914 \\
\hline 4 & Intron 1 & 6602 & $\mathrm{C} / \mathrm{T}$ & & 33 & Exon 5 & 93 & $\mathrm{C} / \mathrm{T}(\mathrm{Thr} 306 \mathrm{Ile})$ & rs272893 \\
\hline 5 & Intron 1 & 6631 & $\mathrm{~A} / \mathrm{G}$ & rs270612 & 34 & Intron 5 & $(1197-1202)$ & ACAACA/del & \\
\hline 6 & Intron 1 & 6790 & $\mathrm{C} / \mathrm{T}$ & & 35 & Intron 5 & (2071-2083) & $(\mathrm{T})_{11-13}$ & \\
\hline 7 & Intron 1 & 14019 & $\mathrm{G} / \mathrm{A}$ & & 36 & Intron 5 & 2282 & $\mathrm{~A} / \mathrm{G}$ & rs272889 \\
\hline 8 & Intron 1 & 14136 & $\mathrm{~T} / \mathrm{C}$ & & 37 & Intron 5 & 2327 & $\mathrm{C} / \mathrm{T}$ & rs272888 \\
\hline 9 & Intron 1 & 14266 & $\mathrm{G} / \mathrm{T}$ & & 38 & Intron 5 & 2617 & G/A & rs272887 \\
\hline 10 & Intron 1 & 14412 & $\mathrm{C} / \mathrm{T}$ & & 39 & Intron 5 & 2744 & $\mathrm{~T} / \mathrm{C}$ & rs272886 \\
\hline 11 & Intron 1 & 15776 & $\mathrm{G} / \mathrm{A}$ & & 40 & Intron 5 & 2781 & $\mathrm{G} / \mathrm{A}$ & \\
\hline 12 & Intron 1 & 15817 & $\mathrm{~A} / \mathrm{G}$ & & 41 & Intron 5 & 4257 & $\mathrm{~A} / \mathrm{G}$ & rs273909 \\
\hline 13 & Intron 1 & 15889 & $\mathrm{G} / \mathrm{A}$ & & 42 & Intron 6 & 5 & G/A & rs2304081 \\
\hline 14 & Intron 1 & 16063 & $\mathrm{~A} / \mathrm{G}$ & & 43 & Intron 6 & (882-917) & $(\mathrm{AC})_{15-18}$ & \\
\hline 15 & Intron 2 & 1105 & $\mathrm{C} / \mathrm{A}$ & & 44 & Intron 6 & 924 & $\mathrm{~A} / \mathrm{C}$ & \\
\hline 16 & Intron 2 & 1229 & $\mathrm{~A} / \mathrm{G}$ & rs270607 & 45 & Intron 6 & 1111 & $\mathrm{G} / \mathrm{C}$ & rs272884 \\
\hline 17 & Intron 2 & 1265 & $\mathrm{G} / \mathrm{A}$ & rs2073838 & 46 & Intron 6 & 1155 & G/A & rs272883 \\
\hline 18 & Intron 3 & 784 & $\mathrm{C} / \mathrm{T}$ & rs2073839 & 47 & Exon 7 & 136 & C/G(Thr394Thr) & rs272879 \\
\hline 19 & Intron 3 & 1022 & $\mathrm{~T} / \mathrm{C}$ & & 48 & Intron 7 & 511 & $\mathrm{~T} / \mathrm{C}$ & \\
\hline 20 & Intron 3 & 1217 & $\mathrm{G} / \mathrm{A}$ & & 49 & Exon 8 & 124 & G/A(Gly462Glu) & \\
\hline 21 & Intron 3 & 1406 & $\mathrm{~A} / \mathrm{G}$ & rs270606 & 50 & Intron 8 & 76 & $\mathrm{C} / \mathrm{T}$ & rs272878 \\
\hline 22 & Intron 3 & 1596 & $\mathrm{G} / \mathrm{A}$ & & 51 & Intron 8 & 3514 & G/A & \\
\hline 23 & Intron 3 & 1720 & $\mathrm{G} / \mathrm{A}$ & & 52 & Intron 8 & 3715 & $\mathrm{G} / \mathrm{A}$ & rs272873 \\
\hline 24 & Intron 3 & 2104 & G/A & & 53 & Intron 8 & 3902 & $\mathrm{~T} / \mathrm{C}$ & \\
\hline 25 & Intron 3 & 7056 & $\mathrm{~A} / \mathrm{G}$ & rs272842 & 54 & Intron 8 & (4064-4089) & $(\mathrm{T})_{18-26}$ & \\
\hline 26 & Intron 3 & 7239 & $\mathrm{~T} / \mathrm{C}$ & rs 270602 & 55 & Intron 8 & 4171 & $\mathrm{G} / \mathrm{A}$ & rs272872 \\
\hline 27 & Intron 3 & 7536 & $\mathrm{~T} / \mathrm{C}$ & rs270601 & 56 & Intron 8 & 4288 & $\mathrm{C} / \mathrm{T}$ & rs2306772 \\
\hline 28 & Intron 3 & 8323 & $\mathrm{G} / \mathrm{C}$ & & 57 & 3' Flanking & 115 & $\mathrm{~T} / \mathrm{A}$ & \\
\hline 29 & Intron 4 & 926 & $\mathrm{G} / \mathrm{T}$ & & 58 & 3' Flanking & 1177 & G/A & rs272867 \\
\hline
\end{tabular}

SLC22A4, Solute carrier family 22, member 4

${ }^{a}$ For SNPs in the $5^{\prime}$ flanking region, intron, or $3^{\prime}$ flanking region, nucleotide positions are counted from the first intronic nucleotide at the exon/ intron junction (for SNPs in the exon, nucleotide positions are counted from the first exonic nucleotide at the exon/intron junction)

Table 3f. Summary of genetic variations detected in the SLC22A5 gene

\begin{tabular}{|c|c|c|c|c|c|c|c|c|c|}
\hline No. & Location & Position $^{\mathrm{a}}$ & Genetic variation & NCBI SNP ID & No. & Location & Position $^{\mathrm{a}}$ & Genetic variation & NCBI SNP ID \\
\hline 1 & 5' Flanking & -225 & $\mathrm{C} / \mathrm{T}$ & & 21 & Intron 2 & 608 & $\mathrm{C} / \mathrm{A}$ & \\
\hline 2 & 5' Flanking & -178 & $\mathrm{G} / \mathrm{C}$ & rs2631369 & 22 & Intron 2 & 2733 & $\mathrm{C} / \mathrm{G}$ & rs183898 \\
\hline 3 & $5^{\prime}$ Flanking & -147 & $\mathrm{~T} / \mathrm{G}$ & rs 2631368 & 23 & Intron 2 & 4037 & $\mathrm{C} / \mathrm{G}$ & rs 274562 \\
\hline 4 & 5' Flanking & -124 & $\mathrm{G} / \mathrm{T}$ & & 24 & Intron 2 & 4370 & $\mathrm{G} / \mathrm{A}$ & \\
\hline 5 & $5^{\prime}$ Flanking & -13 & $\mathrm{C} / \mathrm{G}$ & & 25 & Intron 3 & 77 & $\mathrm{G} / \mathrm{A}$ & rs274559 \\
\hline 6 & Exon 1 & 506 & C/T(Leu95Leu) & rs2631365 & 26 & Intron 3 & 270 & $\mathrm{~A} / \mathrm{G}$ & rs635620 \\
\hline 7 & Intron 1 & 232 & $\mathrm{G} / \mathrm{A}$ & & 27 & Intron 3 & 272 & $\mathrm{G} / \mathrm{A}$ & rs635619 \\
\hline 8 & Intron 1 & 314 & $\mathrm{C} / \mathrm{T}$ & & 28 & Exon 4 & 155 & G/A(Leu269Leu) & rs274558 \\
\hline 9 & Intron 1 & 1048 & G/A & rs 2631363 & 29 & Intron 4 & 13 & $\mathrm{C} / \mathrm{T}$ & rs274557 \\
\hline 10 & Intron 1 & 1246 & $\mathrm{~A} / \mathrm{G}$ & rs 2631362 & 30 & Intron 5 & 445 & $\mathrm{~T} / \mathrm{C}$ & rs2073643 \\
\hline 11 & Intron 1 & 1333 & $\mathrm{~A} / \mathrm{C}$ & rs2631361 & 31 & Intron 5 & 446 & $\mathrm{~T} / \mathrm{A}$ & rs2073644 \\
\hline 12 & Intron 1 & 1845 & $\mathrm{C} / \mathrm{T}$ & rs2631359 & 32 & Intron 5 & 969 & $\mathrm{C} / \mathrm{T}$ & \\
\hline 13 & Intron 1 & 2488 & $\mathrm{C} / \mathrm{A}$ & rs671473 & 33 & Intron 6 & 101 & $\mathrm{~A} / \mathrm{G}$ & rs2073645 \\
\hline 14 & Intron 1 & 5055 & $\mathrm{G} / \mathrm{A}$ & & 34 & Intron 6 & 1119 & $\mathrm{C} / \mathrm{G}$ & rs 274553 \\
\hline 15 & Intron 1 & 6080 & $\mathrm{~A} / \mathrm{G}$ & rs274571 & 35 & Intron 7 & 1084 & $\mathrm{C} / \mathrm{T}$ & rs 274551 \\
\hline 16 & Intron 1 & 6437 & $\mathrm{G} / \mathrm{C}$ & & 36 & Intron 8 & 812 & $\mathrm{C} / \mathrm{A}$ & rs274549 \\
\hline 17 & Intron 1 & 7181 & $\mathrm{C} / \mathrm{T}$ & rs 274570 & 37 & Intron 9 & 188 & $\mathrm{~T} / \mathrm{C}$ & rs2074610 \\
\hline 18 & Intron 1 & 7727 & $\mathrm{C} / \mathrm{T}$ & rs2073642 & 38 & Exon 10 & 931 & $\mathrm{~T} / \mathrm{C}\left(3^{\prime} \mathrm{UTR}\right)$ & rs274548 \\
\hline 19 & Intron 2 & $(173-174)$ & $\mathrm{TC} / \mathrm{del}$ & & 39 & Exon 10 & $(1028-1044)$ & $(\mathrm{T})_{16-18}\left(3^{\prime}\right.$ UTR $)$ & \\
\hline 20 & Intron 2 & 239 & $\mathrm{~T} / \mathrm{C}$ & rs274567 & 40 & Exon 10 & 1428 & T/A(3'UTR) & rs 274547 \\
\hline
\end{tabular}

SLC22A5, Solute carrier family 22, member 5

${ }^{a}$ For SNPs in the 5' flanking region, intron, or 3' flanking region, nucleotide positions are counted from the first intronic nucleotide at the exon/ intron junction (for SNPs in the exon, nucleotide positions are counted from the first exonic nucleotide at the exon/intron junction) 
Table 4. Number and regions of SNPs detected in six $S L C$ genes

\begin{tabular}{|c|c|c|c|c|c|c|c|c|}
\hline \multirow[b]{3}{*}{ Gene } & \multirow[b]{3}{*}{ 5' Flanking } & \multirow[b]{3}{*}{ Intron } & \multirow[b]{3}{*}{ 3' Flanking } & \multicolumn{4}{|l|}{ Exon } & \multirow[b]{3}{*}{ Total } \\
\hline & & & & \multirow[b]{2}{*}{ 5' UTR } & \multicolumn{2}{|l|}{ Coding } & \multirow[b]{2}{*}{ 3' UTR } & \\
\hline & & & & & Nonsynonymous & Synonymous & & \\
\hline SLC10A1 & 0 & 11 & 1 & 0 & 1 & 1 & 0 & 14 \\
\hline SLC15A1 & 0 & 43 & 1 & 1 & 3 & 1 & 2 & 51 \\
\hline$S L C 22 A 1$ & 0 & 22 & 2 & 0 & 4 & 1 & 0 & 29 \\
\hline$S L C 22 A 2$ & 0 & 21 & 2 & 0 & 0 & 2 & 2 & 27 \\
\hline$S L C 22 A 4$ & 3 & 46 & 2 & 0 & 2 & 1 & 0 & 54 \\
\hline$S L C 22 A 5$ & 5 & 29 & 0 & 0 & 0 & 2 & 2 & 38 \\
\hline Total & 8 & 172 & 8 & 1 & 10 & 8 & 6 & 213 \\
\hline
\end{tabular}

UTR, untranslated region

Table 5. Novel SNPs detected in exons of six $S L C$ genes

\begin{tabular}{lllll}
\hline Region & Gene & Location & Position & SNP \\
\hline 5' UTR & $S L C 15 A 1$ & Exon 1 & 25 & $\mathrm{C} / \mathrm{T}$ \\
Coding & & & \\
$\quad$ Nonsynonymous & $S L C 15 A 1$ & Exon 16 & 107 & G/C(Gly419Ala) \\
& $S L C 22 A 1$ & Exon 5 & 9 & C/T(Pro283Leu) \\
& & Exon 5 & 20 & C/G(Arg287Gly) \\
Synonymous & $S L C 22 A 4$ & Exon 8 & 124 & G/A(Gly462Glu) \\
3' UTR & $S L C 10 A 1$ & Exon 1 & 307 & G/A(Thr75Thr) \\
& $S L C 15 A 1$ & Exon 23 & 790 & A/T \\
& $S L C 22 A 2$ & Exon 11 & 427 & G/A \\
\hline
\end{tabular}

\section{References}

Adibi SA (1997) The oligopeptide transporter (Pept-1) in human intestine: biology and function. Gastroenterology 113:332-340

Burckhardt G, Wolff NA (2000) Structure of renal organic anion and cation transporters. Am J Physiol Renal Physiol 278:F853-F866

Burwinkel B, Kreuder J, Schweitzer S, Vorgerd M, Gempel K, Gerbitz K-D, Kilimann MW (1999) Carnitine transporter OCTN2 mutations in systemic primary carnitine deficiency: a novel Arg169Gln mutation and a recurrent Arg282ter mutation associated with an unconventional splicing abnormality. Biochem Biophys Res Commun 261:484-487

Covitz K-MY, Amidon GL, Sadée W (1998) Membrane topology of the human dipeptide transporter, hPEPT1, determined by epitope insertions. Biochemistry 37:15214-15221

Craddock A, Love M, Daniel R, Kirby L, Walters H, Wong M, Dawson P (1998) Expression and transport properties of the human ileal and renal sodium-dependent bile acid transporter. Am J Physiol 274:G157-G169

Gartung C, Ananthanarayanan M, Rahman MA, Schuele S, Nundy S, Soroka CJ, Stolz A, Suchy FJ, Boyer JL (1996) Down-regulation of expression and function of the rat liver $\mathrm{Na}^{+} /$bile acid cotransporter in extrahepatic cholestasis. Gastroenterology 110:199-209

Gorboulev V, Ulzheimer JC, Akhoundova A, Ulzheimer-Teuber I, Karbach U, Quester S, Baumann C, Lang F, Busch AE, Koepsell $\mathrm{H}$ (1997) Cloning and characterization of two human polyspecific organic cation transporters. DNA Cell Biol 16:871-881

Green RM, Gollan JL, Hagenbuch B, Meier PJ, Beier DR (1997) Regulation of hepatocyte bile salt transporters during hepatic regeneration. Am J Physiol 273:G621-G627

Iida A, Sekine A, Saito S, Kitamura Y, Kitamoto T, Osawa S, Mishima C, Nakamura Y (2001) Catalog of 320 single nucleotide polymorphisms (SNPs) in 20 quinone oxidoreductase and sulfotransferase genes. J Hum Genet 46:225-240

Koepsell H (1998) Organic cation transporters in intestine, kidney, liver, and brain. Annu Rev Physiol 60:243-266
Lamhonwah A-M, Tein I (1998) Carnitine uptake defect: frameshift mutations in the human plasmalemmal carnitine transporter gene. Biochem Biophys Res Commun 252:396-401

Leibach FH, Ganapathy V (1996) Peptide transporters in the intestine and the kidney. Annu Rev Nutr 16:99-119

Liang R, Fei Y-J, Prasad PD, Ramamoorthy S, Han H, Yang-Feng TL, Hediger MA, Ganapathy V, Leibach FH (1995) Human intestinal $\mathrm{H}^{+} /$peptide cotransporter: cloning, functional expression, and chromosomal localization. J Biol Chem 270:6456-6463

Meier PJ (1995) Molecular mechanisms of hepatic bile salt transport from sinusoidal blood into bile. Am J Physiol 269:G801-G812

Muller M, Jansen PLM (1997) Molecular aspects of hepatobiliary transport. Am J Physiol 272:G1285-G1303

Nezu J, Tamai I, Oku A, Ohashi R, Yabuuchi H, Hashimoto N, Nikaido H, Sai Y, Koizumi A, Shoji Y, Takada G, Matsuishi T, Yoshino M, Kato H, Ohura T, Tsujimoto G, Hayakawa J, Shimane M, Tsuji A (1999) Primary systemic carnitine deficiency is caused by mutations in a gene encoding sodium ion-dependent carnitine transporter. Nat Genet 21:91-94

Nickerson DA, Tobe VO, Taylor SL (1997) PolyPhred: automating the detection and genotyping of single nucleotide substitutions using fluorescence-based resequencing. Nucleic Acids Res 25:27452751

Saito S, Iida A, Sekine A, Eguchi C, Miura Y, Nakamura Y (2001) Seventy genetic variations in human microsomal and soluble epoxide hydrolase genes (EPHX1 and EPHX2) in the Japanese population. J Hum Genet 46:325-329

Sekine A, Saito S, Iida A, Mitsunobu Y, Higuchi S, Harigae S, Nakamura Y (2001) Identification of single-nucleotide polymorphisms (SNPs) of human $N$-acetyltransferase genes NAT1, NAT2, $A A N A T, A R D 1$, and L1CAM in the Japanese population. J Hum Genet 46:314-319

Shiao T, Iwahashi M, Fortune J, Quattrochi L, Bowman S, Wick M, Qadri I, Simon FR (2000) Structural and functional characterization of liver cell-specific activity of the human sodium/taurocholate cotransporter. Genomics 69:203-213

Tamai I, Yabuuchi H, Nezu J, Sai Y, Oku A, Shimane M, Tsuji A (1997) Cloning and characterization of a novel human $\mathrm{pH}-$ 
dependent organic cation transporter, OCTN1. FEBS Lett 419:107111

Tamai I, Ohashi R, Nezu J, Yabuuchi H, Oku A, Shimane M, Sai Y, Tsuji A (1998) Molecular and functional identification of sodium ion-dependent, high affinity human carnitine transporter OCTN2. J Biol Chem 273:20378-20382

Tang NLS, Ganapathy V, Wu X, Hui J, Seth P, Yuen PMP, Fok TF, Hjelm NM (1999) Mutations of OCTN2, an organic cation/carnitine transporter, lead to deficient cellular carnitine uptake in primary carnitine deficiency. Hum Mol Genet 8:655-660

Vaz FM, Scholte HR, Ruiter J, Hussaarts-Odijk LM, Rodrigues Pereira R, Schweitzer S, de Klerk JBC, Waterham HR, Wanders RJA (1999) Identification of two novel mutations in OCTN2 of three patients with systemic carnitine deficiency. Hum Genet 105:157-161

Wang Y, Ye J, Ganapathy V, Longo N (1999) Mutations in the organic cation/carnitine transporter OCTN2 in primary carnitine deficiency. Proc Natl Acad Sci 96:2356-2360
Wang Y, Taroni F, Garavaglia B, Longo N (2000) Functional analysis of mutations in the OCTN2 transporter causing primary carnitine deficiency: lack of genotype-phenotype correlation. Hum Mutat 16:401-407

Wang Y, Korman SH, Ye J, Gargus JJ, Gutman A, Taroni F, Garavaglia B, Longo N (2001) Phenotype and genotype variation in primary carnitine deficiency. Genet Med 3:387-392

Yabuuchi H, Tamai I, Nezu J, Sakamoto K, Oku A, Shimane M, Sai Y, Tsuji A (1999) Novel membrane transporter OCTN1 mediated multispecific, bi-directional and $\mathrm{pH}$-dependent transport of organic cations. J Pharmacol Exp Ther 289:768-773

Zhang L, Dresser MJ, Gray AT, Yost SC, Terashita S, Giacomini KM (1997) Cloning and functional expression of a human liver organic cation transporter. Mol Pharmacol 51:913-921

Zhang L, Brett CM, Giacomini KM (1998) Role of organic cation transporters in drug absorption and elimination. Annu Rev Pharmacol Toxicol 38:431-460 